Cerebrovasc Dis 2007;24:316-317

DOI: $10.1159 / 000106518$

\section{Epilepsy in Cerebral Autosomal Dominant Arteriopathy with Subcortical Infarcts and Leukoencephalopathy}

\section{Joost Haan a, c, Saskia A.J. Lesnik Oberstein ${ }^{\text {b }}$, Michel D. Ferrari ${ }^{\text {a }}$}

Departments of a Neurology and ${ }^{b}$ Clinical Genetics, Center for Human and Clinical Genetics, Leiden University Medical Center, Leiden, and 'Department of Neurology, Rijnland Hospital, Leiderdorp, The Netherlands

\section{Introduction}

Cerebral autosomal dominant arteriopathy with subcortical infarcts and leukoencephalopathy (CADASIL) is mainly characterized by recurrent transient ischemic attacks (TIAs), strokes and vascular dementia $[1,2]$. Migraine with aura occurs in $\pm 40 \%$ of patients and psychiatric disturbances in $\pm 30 \%$. Affected individuals have prominent and characteristic abnormalities on brain MRI, increasing with age [3]. Mutations in the NOTCH3 gene cause CADASIL.

In general, the overall incidence of seizures related to ischemic stroke was found to be around 9\% [4]. Epilepsy can also be a consequence of subcortical infarcts [5]. The frequency of epilepsy in large series of CADASIL patients is approximately $10 \%$, mostly occurring as a late symptom $[1,2,6,7]$. Rarely, patients have been described with epilepsy early in the course of their disease $[6,8]$. Here, we report a patient with epilepsy as a prominent and early symptom, occurring years before the symptoms became more typical and a diagnosis of CADASIL was made.

\section{Case Report}

In 1995, a then 42 -year-old female (II-5 in the pedigree) presented in another hospital with 2 unprovoked generalized tonicclonic epileptic seizures (fig. 1). Her medical history consisted of an anxiety disorder with panic attacks, for which she was hospitalized several times and for which she took alprazolam at the time of the epileptic seizures. The panic attacks started as agoraphobia but later worsened into a fear of being alone. Based on the description of these episodes it was unlikely that they represented ictal fear episodes as earlier manifestation of epilepsy, but this cannot be completely ruled out. Neurological examination at presentation was unremarkable. Two routine EEGs were unremarkable, a third showed bilateral temporal sharp wave activity. A cerebral MRI revealed widespread white matter lesions in both hemispheres (without pathological enhancement), and the radiologist suggested multiple sclerosis as a possible diagnosis (fig. 2). CSF analysis showed normal cell count and protein, and no oligoclonal bands. An extensive analysis for various other causes of white matter lesions gave normal results, including cardiac echography, carotid ultrasound, determination of lactate, pyruvate, very long chain fatty acids, thyroid function, HIV, TPHA, vitamins, lysozyme, ACE, and levels of arylsulfatase A and galactocerebrosidase. Homocysteine levels after methionine increased from 7.4 to $53.5 \mu \mathrm{mol} / \mathrm{l}$ and the patient was treated with folate and vitamin B6. Several interictal EEGs were normal. Neuropsychological testing in 1995 revealed an IQ of 106 (verbal 110, performal

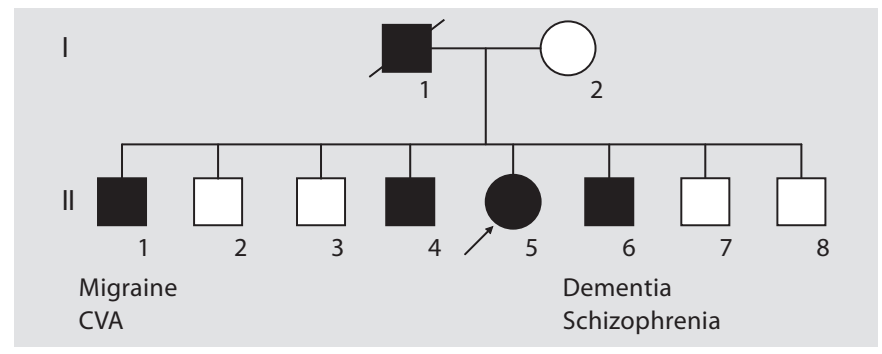

Fig. 1. Pedigree. Square $=$ Male; circle $=$ female; filled $=$ affected; arrow $=$ proband CVA = cerebrovascular accident.

100), which was normal in the context of her level of education. A second neuropsychological investigation in 1997 showed impaired memory and recall, and abnormal calculation. A repeated cerebral MRI in 1998 was unchanged. The patient had lost contact with her family and was uncertain about a family history of neurological diseases, including epilepsy and stroke. Because of repeated generalized tonic-clonic seizures, she was treated with carbamazepine, which stopped the attacks. As soon as the dose was decreased, attacks reoccurred, also after cessation of alprazolam.

In 2000 the patient's older brother (II-4) visited the outpatient clinic of our hospital because of a TIA in the left hemisphere at the age of 48 . A cerebral MRI showed extensive white matter lesions in both hemispheres. An extensive search for vascular risk factors was negative. In the past the patient had had occasional headaches, without nausea, or photo- and phonophobia. This patient gave a more detailed family history. The father (I-1) had died at the age of 60 years after multiple strokes since his late fifties. At the time of death he was severely demented. One brother (II-1) suffered from migraine (no further details) and had had a stroke at the age of 45. Another brother (II-6) had schizophrenia, diabetes mellitus and cerebral white matter lesions on MRI. Three of the 8 sibs (II-2, II-3 and II-8) were asymptomatic.

Because of the typical clinical and radiological presentation in II-4, a diagnosis of CADASIL was suspected. Three symptomatic family members (II-4, II-5 and II-6) gave consent for NOTCH3 gene analysis. In all 3, a typical CADASIL mutation (Arg182Cys) in exon 4 was found.

\section{Discussion}

The medical history of our proband (II-5) is remarkable for several reasons. Firstly, she did not suffer from typical CADASIL symptoms such as TIAs, infarcts or migraine with aura. In large series, only a small minority of CADASIL patients do not have any of these typical symptoms [6-8]. Secondly, she presented with epileptic seizures, which has been described as a presenting neurological symptom of CADASIL only rarely [6]. Because of this atypical clinical presentation, the diagnosis of CADASIL was - in spite of suggestive abnormalities on cerebral MRI - only considered when her brother developed typical symptoms of the disease. Retrospectively, II-5 had some symptoms more typical of CADASIL, such as cognitive decline, documented by neuropsychological investigations. Also, like her brother (II-6), she had a history of psychiatric disease, which is not rare in CADASIL [9]. Finally, her homocysteine metabolism was abnormal (first interpreted 

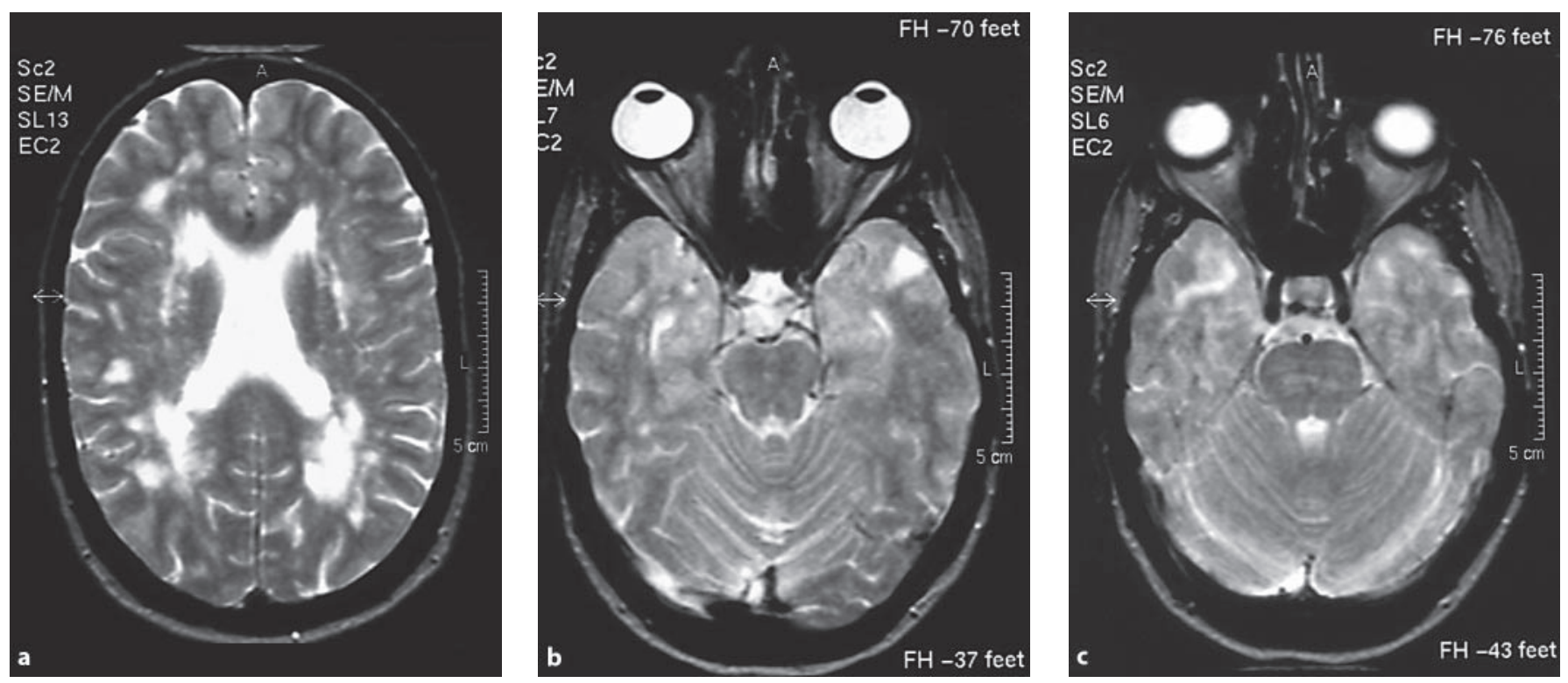

Fig. 2a-c. Cerebral MRI of the proband (II-5), showing typical CADASIL abnormalities, including subcortical infarcts, leukoencephalopathy and involvement of the anterior temporal lobe and external capsule.

as explanation of the white matter lesions), which has also been described in CADASIL [10].

In a series of 102 CADASIL patients, 9 had generalized and 1 had focal epilepsy [6]. A history of previous stroke was present in 9 of 10 epilepsy patients, suggesting a secondary origin of the epilepsy. In none of the patients was epilepsy the initial symptom. The median age at onset of epilepsy was 50 years, with a range of 23-63. Pooled CADASIL data showed that 6 of 105 patients had seizures, of which the type was not further specified [8]. In 3 of 6 this was the initial symptom.

There seems to be no genotype-phenotype correlation between certain NOTCH3 mutations and epilepsy. The most frequent type of mutation in CADASIL is a missense point mutation either creating or deleting 1 cysteine residue [1]. No relation was found between the site of missense mutations and the occurrence of epilepsy [7]. One of 5 patients with a rare inframe deletion causing the loss of 3 cysteine residues had epilepsy, but this could easily be ascribed to chance [11]. Finally, a patient homozygous for an R133C missense mutation had no epilepsy [12].

In conclusion, although in large series approximately $10 \%$ of patients suffer from epilepsy late in the course of their disease, CADASIL can also present with epilepsy. One should be aware of this when encountering a patient with epilepsy and otherwise unexplained white matter lesions on cerebral MRI, especially when there is a family history of TIAs, stroke or migraine with aura.

\section{References}

1 Lesnik Oberstein SAJ, Haan J: CADASIL. Panminerva Med 2004;46: 265-276.

2 Dichgans M: CADASIL: a monogenic condition causing stroke and subcortical vascular dementia. Cerebrovasc Dis 2002;13(suppl 2):3741.
3 Van Den Boom R, Lesnik Oberstein SAJ, Ferrari MD, Haan J, Van Buchem MA: Cerebral autosomal dominant arteriopathy with subcortical infarcts and leukoencephalopathy: MR imaging findings at different ages - 3rd-6th decades. Radiology 2003;229:683-690.

4 De Reuck J, Vanhee F, Van Maele G, Claeys I: Magnetic resonance imaging after seizures in patients with an ischemic stroke. Cerebrovasc Dis 2007;23:339-343.

5 Bentes C, Pimentel J, Ferro JM: Epileptic seizures following subcortical infarcts. Cerebrovasc Dis 2001;12:331-334.

6 Dichgans M, Mayer M, Uttner I, et al: The phenotypic spectrum of CADASIL: clinical findings in 102 cases. Ann Neurol 1998;44:731-739.

7 Singhal S, Bevan S, Barrick T, Rich P, Markus HS: The influence of genetic and cardiovascular risk factors on the CADASL phenotype. Brain 2004;127:2031-2038.

8 Desmond DW, Moroney JT, Lynch T, et al: The natural history of CADASIL: a pooled analysis of previously published cases. Stroke 1999;30: 1230-1233.

9 Lagas PA, Juvonen V: Schizophrenia in a patient with cerebral autosomally dominant arteriopathy with subcortical infarcts and leucoencephalopathy (CADASIL disease). Nord J Psychiatry 2001;55:41-42.

10 Flemming KD, Nguyen TT, Abu-Lebdeh HS, et al: Hyperhomocysteinemia in patients with CADASIL. Mayo Clin Proc 2001;76:1213-1218.

11 Dichgans M, Herzog J, Gasser T: NOTCH3 mutation involving three cysteine residues in a family with typical CADASIL. Neurology 2001; 57:1714-1717.

12 Tuominen S, Juvonen V, Amberla K, et al: Phenotype of a homozygous CADASIL patient in comparison to 9 age-matched patients with the same R133C Notch3 mutation. Stroke 2001;32:1767-1774.

J. Haan, MD, PhD

Department of Neurology (K5Q), Leiden University Medical Center PO Box 9600

NL-2300 RC Leiden (The Netherlands)

Tel. +31 71526 2895, Fax +31 71524 8253, E-Mail jhaan@rijnland.nl 